\section{Xpert MTB/RIF and pulmonary tuberculosis: time to delve deeper?}

\author{
Lancelot M Pinto, ${ }^{1}$ Zarir F Udwadia ${ }^{2}$
}

I find out what the world needs, then proceed to invent it. Thomas Edison.

If Edison, the world's most prolific inventor, had to get around to creating a new diagnostic test for tuberculosis (TB), he would have considered himself pleased with the Xpert MTB/RIF test. Here is the new diagnostic test for TB that the physician in the developing world has been waiting for. It does two things, and does them both well. It diagnoses TB with a higher sensitivity than a sputum smear examination: a single Xpert MTB/RIF test diagnoses $98 \%$ of sputum smear positive cases and around $70 \%$ of smear-negative patients. ${ }^{1}$ More importantly, for the physician practicing in areas with a high prevalence of multi-drug resistant TB (MDR-TB), it diagnoses rifampicin resistance (a surrogate marker for MDR-TB) with around 98\% sensitivity and specificity, thus identifying patients who need second-line drugs. ${ }^{2}$ That it does this with safety, speed and simplicity is what makes this test a 'game changer'. ${ }^{3}$ A result is obtained within $90 \mathrm{~min}$, lopping off 68 weeks from the time that a traditional culture would take to diagnose MDR-TB. Called a 'laboratory in a cartridge', the test comprises of a cartridge-based system using an automated real-time, PCR-based assay with molecular beacon technology. The test, which is easy to learn, can be performed by a technician with 2 days of training. Robust enough to be performed in emergency departments and small laboratories in hospitals across the developing world, it poses substantially less of a biohazard risk and hence obviates the need for bio safety cabinets, which are anyway conspicuous by their absence in these regions. ${ }^{4}$

This test, initially evaluated in sputum samples for pulmonary $\mathrm{TB}$, has been tested with success in a few small studies for diagnosing pulmonary TB from

\footnotetext{
${ }^{1}$ Respiratory Epidemiology \& Clinical Research Unit, Montréal Chest Institute, McGill University, Montréal, Canada; ${ }^{2}$ Department of Respiratory Medicine, P.D. Hinduja National Hospital and Medical Research Centre, Mumbai, India
}

Correspondence to Dr Zarir F Udwadia, Department of Respiratory Medicine, P.D. Hinduja National Hospital and Medical Research Centre, Mahim, Mumbai 400016, India; zariffudwadia@gmail.com bronchoalveolar lavage fluid (BALF). ${ }^{5-8}$ When it comes to extra-pulmonary sites, recent studies from Italy, ${ }^{9}$ Germany ${ }^{10}$ and India ${ }^{11}$ demonstrate the impressive yield of the Xpert MTB/RIF from a variety of extra-pulmonary sites. The Italian study, which represented the largest number of non-respiratory samples tested with the Xpert MTB/RIF to date (1476 samples from 1068 patients), found an impressive overall sensitivity of $81.3 \%$ and specificity of $99.8 \%$. Biopsies, pus, urine and cerebrospinal fluid (CSF) had sensitivities of $>85 \%$, gastric aspirates around $80 \%$ and cavitatory fluids (pleural, ascitic) around $50 \%$. These figures represent considerable advances on the poor yield from traditional cultures.

Despite being superior in accuracy to smear microscopy, the diagnostic performance of Xpert MTB/RIF is likely to be equally dependent on the quality of the sputum specimen provided. Samples with a predominance of saliva are unlikely to bear mycobacteria, and are consequently reported as being negative for $\mathrm{TB}$ when tested with smear microscopy. ${ }^{12} \mathrm{~A}$ recent study from Indonesia reported that of the subjects who submitted sputum smears for examination, only a third could produce at least one good sample. ${ }^{13}$ The inadequacy of good quality sputum specimens could possibly have contributed to the fact that in $2011,44 \%$ of the 4.7 million new cases of pulmonary TB reported to WHO were sputum smear negative. ${ }^{14}$ Thus, in the potentially underdiagnosed population of sputum-scarce individuals with possible TB, the successful use of Xpert MTB/RIF on BALF would be a useful addition to a limited armamentarium of available diagnostic tools. The study by Theron et $a l^{15}$ is the first large prospective study that explores the utility of the test in BALF specimens collected from individuals with possible $\mathrm{TB}$ in a high-HIV burden setting endemic for TB.

The study, conducted in Cape Town, South Africa, enrolled 154 individuals with suspected pulmonary $\mathrm{TB}$ who were referred for bronchoscopy. These individuals had symptoms and radiographic features compatible with TB and either had two consecutive smears that were negative for acid-fast bacilli or were unable to expectorate sputum. In addition to measuring the diagnostic accuracy of the test, the authors attempted to measure the clinical impact of the test by comparing phase I, in which the test was studied in a research milieu, with phase II, in which the results of the test informed clinical practice. Even in this population, in which a majority $(72 \%)$ of included individuals were sputum-scarce and likely to have a lower mycobacterial burden, the Xpert MTB/RIF test demonstrated an overall sensitivity of $93 \%$ (95\% CI $77 \%$ to $98 \%$ ), which was significantly higher than that for smear microscopy $(58 \%, 95 \%$ CI 39\% to $75 \%$ ), with comparable specificities. The performance was similar in persons living with HIV, and was not affected by centrifugation of the BALF. Genotype MTBDRplus assay performed on five of the 11 false-positive Xpert MTB/RIF results from the study (with culture as the reference standard) in which samples were available suggested these results to be true positives, possibly indicating inaccuracies in the reference standard. The median time-to-detection for smear-negative individuals with $\mathrm{TB}$ was shortened from 29 days (IQR 18,41) to 0 days (IQR 0,0), and the percentage of individuals on empiric TB treatment (those presumptively treated on clinical and radiological grounds without definitive laboratory evidence of disease) decreased from $79 \%$ in phase I to $20 \%$ in phase II. However, the use of the Xpert MTB/RIF test neither significantly impacted the overall proportion of patients who were initiated on anti-TB treatment nor did it significantly change the time to treatment initiation. This was attributed to a high proportion (79\%) of all treated individuals being treated empirically in phase I, only a quarter of whom were later proven to have TB.

The individuals in this large, prospective study were well characterised and thoroughly investigated, and the conduct of the study in two phases allowed for comparisons in the clinical impact of the test. As rightly pointed out by the authors, there is a need for assessing the true clinical impact in a real-world setting before implementing tests based on the assumption that improved accuracy in a research setting translates into a significant positive clinical impact. This is especially true for a test such as a bronchoscopy and the collection of BALF, which is invasive, expensive and likely to be only offered in tertiary care institutes in the developing world. The gain in the lowering of biohazard risks from the use of Xpert MTB/RIF may also be offset by the increased risk to healthcare workers and patients from such 
procedures, which are known to generate infectious aerosols. ${ }^{16}$ The cost of the use of Xpert MTB/RIF is also a crucial aspect in the assessment of the feasibility of the use of this technology, especially in the developing world. The steady decline in the cost of each cartridge has been encouraging. The cost per cartridge is currently pegged at $\$ 9.98$, half that of its initial cost of $\$ 18.68$ when this test was first endorsed by WHO in $2010 .^{17}$ The test has been proven to be cost-effective, but this does not necessarily denote affordability. For example, providing the test to just $15 \%$ of individuals with possible $\mathrm{TB}$ in India would consume the entire annual TB budget of the Indian Revised National TB Control Program. ${ }^{18}$ An easier, relatively inexpensive and more accessible procedure such as the use of sputum induction techniques has been reported to consistently improve the performance of smear microscopy, ${ }^{19}$ but was not investigated by the authors in the study, and is a potential target for future diagnostic research.

In conclusion, in a high-HIV burden setting endemic for TB, Xpert MTB/RIF, when performed on BALF specimens, significantly outperforms smear microscopy in individuals with possible TB who have scarce sputum. However, whether such an enhancement in performance characteristics of a diagnostic test for pulmonary TB results in better clinical outcomes in a cost-effective and affordable manner and in comparison with alternative strategies such as those employing the use of induced sputum remains unproven, and should be the focus of future research.

Competing interests None.
Provenance and peer review Not commissioned; internally peer reviewed.

To cite Pinto LM, Udwadia ZF. Thorax 2013;68: 987-988.

Published Online First 4 July 2013

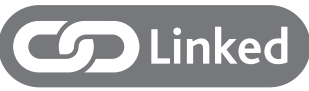

http://dx.doi.org/10.1136/thoraxjnl-2013-203485

Thorax 2013;68:987-988.

doi:10.1136/thoraxjn-2013-203885

\section{REFERENCES}

1 Boehme CC, Nabeta P, Hillemann D, et al. Rapid molecular detection of tuberculosis and rifampin resistance. N Engl J Med 2010;363:1005-15.

2 Helb $\mathrm{D}$, Jones $\mathrm{M}$, Story $\mathrm{E}$, et al. Rapid detection of Mycobacterium tuberculosis and rifampin resistance by use of on-demand, near-patient technology. J Clin Microbiol 2010;48:229-37.

3 Evans CA. GeneXpert-a game-changer for tuberculosis control? PLoS Med 2011;8:e1001064.

4 Boehme CC, Nicol MP, Nabeta P, et al. Feasibility, diagnostic accuracy, and effectiveness of decentralised use of the Xpert MTB/RIF test for diagnosis of tuberculosis and multidrug resistance: a multicentre implementation study. Lancet 2011;377:1495-505

5 Teo J, Jureen R, Chiang D, et al. Comparison of two nucleic acid amplification assays, the Xpert MTB/RIF assay and the amplified Mycobacterium Tuberculosis Direct assay, for detection of Mycobacterium tuberculosis in respiratory and nonrespiratory specimens. J Clin Microbiol 2011;49:3659-62.

6 Armand S, Vanhuls P, Delcroix G, et al. Comparison of the Xpert MTB/RIF test with an IS6110-TaqMan real-time PCR assay for direct detection of Mycobacterium tuberculosis in respiratory and nonrespiratory specimens. J Clin Microbiol 2011:49:1772-6.

7 Miller MB, Popowitch EB, Backlund MG, et al. Performance of Xpert MTB/RIF RUO assay and IS6110 real-time PCR for Mycobacterium tuberculosis detection in clinical samples. J Clin Microbiol 2011;49:3458-62.
8 Zeka AN, Tasbakan S, Cavusoglu C. Evaluation of the GeneXpert MTB/RIF assay for rapid diagnosis of tuberculosis and detection of rifampin resistance in pulmonary and extrapulmonary specimens. J Clin Microbiol 2011:49:4138-41.

9 Tortoli E, Russo C, Piersimoni C, et al. Clinical validation of Xpert MTB/RIF for the diagnosis of extrapulmonary tuberculosis. Eur Respir $\mathrm{J}$ 2012:40:442-7

10 Hillemann D, Rusch-Gerdes S, Boehme C, et al. Rapid molecular detection of extrapulmonary tuberculosis by the automated GeneXpert MTB/RIF system. J Clin Microbiol 2011;49: 1202-5

11 Vadwai V, Boehme C, Nabeta P, et al. Xpert MTB/RIF: a new pillar in diagnosis of extrapulmonary tuberculosis? J Clin Microbiol 2011:49:2540-5.

12 Hepple P, Nguele P, Greig J, et al. Direct microscopy versus sputum cytology analysis and bleach sedimentation for diagnosis of tuberculosis: a prospective diagnostic study. BMC Infect Dis 2010;10:276.

13 Sakundarno $M$, Nurjazuli N, Jati SP, et al. Insufficient quality of sputum submitted for tuberculosis diagnosis and associated factors, in Klaten district, Indonesia. BMC Pulm Med 2009;9:16.

14 World Health Organization. Global Tuberculosis Report 2012.WHO/HTM/TB/20126 2012.

15 Theron G, Peter J, Meldau R, et al. Accuracy and impact of Xpert MTB/RIF for the diagnosis of smear-negative or sputum-scarce tuberculosis using bronchoalveolar lavage fluid. Thorax 2013:68:1043-51.

16 Nelson KE, Larson PA, Schraufnagel DE, et al. Transmission of tuberculosis by flexible fiberbronchoscopes. Am Rev Respir Dis 1983:127:97-100.

17 Lawn SD, Mwaba P, Bates M, et al. Advances in tuberculosis diagnostics: the Xpert MTB/RIF assay and future prospects for a point-of-care test. Lancet Infect Dis 2013;13:349-61.

18 Dowdy DW, Cattamanchi A, Steingart KR, et al. Is scale-up worth it? Challenges in economic analysis of diagnostic tests for tuberculosis. PLoS Med 2011;8:e1001063.

19 Hepple P, Ford N, McNerney R. Microscopy compared to culture for the diagnosis of tuberculosis in induced sputum samples: a systematic review. Int $J$ Tuberc Lung Dis 2012;16:579-88. 